Giacomo Giacobini

Université de Turin, Italie

Musée d'Anatomie

Cristina Cilli

Pôle muséal de Turin

Giancarla Malerba

Pôle muséal de Turin

Musée d'Anatomie de Turin

\title{
Que faire des musées de savants? Le défi du Musée d'Anatomie de Turin
}

"Des musées savants aux musées communicants", cette phrase introduisant le dossier de ce numéro de la revue Hermès, convient parfaitement au Musée d'Anatomie humaine de l'Université de Turin, à son histoire et au projet de rénovation qui a conduit à son ouverture au public en 2007. Le musée a été créé par des savants et pour des savants, pour conserver les produits d'une anatomie traditionnelle - préparations et modèles - qui se sont accumulés pendant presque deux cents ans. Lieu de mémoire d'une école scientifique ancienne et prestigieuse, pour des raisons liées à l'évolution des sujets de recherche, le musée a perdu au début du $\mathrm{xx}^{\mathrm{e}}$ siècle tout intérêt en devenant une institution obsolète ${ }^{1}$.

Immobile dans ses collections qui n'étaient plus enrichies par la recherche, devenu inutile pour l'enseignement, le musée sombra progressivement, et en définitive "heureusement», dans l'oubli. La présence d'autres vastes locaux dans le même bâtiment lui permit de résister miraculeusement à la pression de l'institution qui, notamment dans les années 1960 et 1970, avait besoin d'aménager de nouveaux laboratoires ou de nouvelles salles de cours. Il traversa le $\mathrm{xx}^{\mathrm{e}}$ siècle comme cristallisé pour faire 
finalement l'objet, au début du $\mathrm{xxI}^{\mathrm{e}}$, d'un projet de restauration associé à une réflexion sur ses potentialités de communication en direction du grand public.

\section{Histoire des collections, du musée et du bâtiment}

Le Musée académique des Sciences créé en 1739 dans le palais de l'Université, aujourd'hui siège de sa présidence, prévoyait une salle d'anatomie. Le projet du musée ayant été approuvé par le roi Charles-Emmanuel III $^{2}$, les différentes collections donnèrent lieu successivement à la création de musées indépendants sous la direction de grands savants de l'époque, soucieux de développer leur discipline et pour cela de conserver les traces de leurs travaux, mais aussi d'en assurer la visibilité au sein de l'establishment académique. Après la Restauration, le musée d'anatomie s'enrichit abondamment grâce à l'activité de l'illustre anatomiste Luigi Rolando (1773-1831) auquel le musée est dédié. Une importante collection de modèles en cire fut réunie par lui et par ses successeurs ${ }^{3}$. Au cours de la seconde moitié du XIX $\mathrm{X}^{\mathrm{e}}$ siècle, à ces collec tions d'anatomie «artificielle» s'ajoutèrent des séries de préparations d'anatomie «naturelle» séchées ou conservées dans des liquides. Le musée possède également des collections d'intérêt anthropologique, phrénologique, primatologique, embryologique et artistique, ainsi que des collections d'instruments anciens. Il recèle enfin un fonds de documents et de photographies et une bibliothèque historique.

$\mathrm{Au}$ cours de son histoire, le Musée d'Anatomie a subi au moins quatre déplacements, le dernier en 1898 dans le Palais des Instituts anatomiques construit pour être le siège des Instituts d'anatomie humaine normale, d'anatomie pathologique et de médecine légale. Le bâtiment, monumental, constitue un exemple remarquable d'architecture scientifique de l'époque; il souligne l'importance de la discipline et le prestige de l'école anatomique turinoise à la fin $\mathrm{du} \mathrm{XIX}^{\mathrm{e}}$ siècle. Les salles du Musée d'Anatomie évoquent une cathédrale, avec trois nefs supportées par des séries de colonnes en granit et des sortes de chapelles latérales créées par les vitrines, chacune étant surmontée par le portraità l'huile sur toile d'un personnage important pour l'histoire de l'anatomie, de vrais «saints de la science». La seconde salle est éclairée par un vitrail représentant des sections du cerveau. L'ensemble du dispositif architectural contribue à créer l'impression d'un temple de la science, conformément à l'importance attribuée à cette dernière dans la société à l'époque du positivisme (Avataneo et Montaldo, 2003).

\section{Le projet de restauration et de communication}

Puisquau cours du $\mathrm{xx}^{\mathrm{e}}$ siècle le musée n'a pas subi d'importantes modifications, il offre aujourd'hui l'opportunité de visiter un exemple rare de musée scientifique du XIX ${ }^{\mathrm{e}}$ siècle resté presque intact dans des locaux construits exprès pour lui. Il a été récemment restauré: le projet comportait la restauration des locaux, des tableaux, des vitrines, et des collections (modèles en cire, en papier mâché, en bois et ivoire, et préparations anatomiques).

L'équipe chargée de la rénovation (comité scientifique, direction du projet, architecte designer) a considéré que pour cette institution - constituant un témoignage du musée scientifique au $\mathrm{XIX}^{\mathrm{e}}$ siècle où le temps semble s'être arrêté - les opérations de restauration devaient souligner son prestige historique, architectural, artistique et s'efforcer de retrouver l'atmosphère de l'époque. Ces prin- 
cipes ont été décidés comme prioritaires dans les interventions de restauration et de valorisation. La muséographie originale a été conservée, en dépit des problèmes de communication scientifique qu'elle pose. De fait, les vitrines sans éclairage interne, regorgent d'objets. Elles contiennent aussi très peu de textes explicatifs, ce qui était normal dans les musées d'alors. Une attention spéciale a dû être attribuée à l'étude de stratégies pour la réinterprétation du musée, pour sa transformation en musée communicant, tout en sauvegardant le charme et l'intérêt d'une mise en scène scientifique et savante du $\mathrm{XIX}^{\mathrm{e}}$ siècle. Cette réflexion, menée en même temps que les opérations de restauration, a été poursuivie après l'ouverture au public.

Le musée n'est pas resté muet comme il l'était à l'origine; il est maintenant devenu à même de nous raconter l'histoire des collections, de parler de découvertes scientifiques, d'évoquer des événements liés aux activités de personnalités qui ont fait partie de l'école anatomique turinoise au cours des derniers trois siècles et qui ont parfois aussi joué un rôle dans des événements non scientifiques de l'histoire de cette ville, longtemps capitale politique et culturelle. Une de ces personnalités est l'anatomiste Luigi Rolando, dédicataire du musée. Il fut, au début du XIX ${ }^{\mathrm{e}}$ siècle, l'un des chercheurs qui contribuèrent de manière significative à la connaissance de l'anatomie cérébrale (Caputi et al., 1995; Dini, 2001).

Les visiteurs sont invités à découvrir les collections du musée en utilisant soit une plaquette présentant synthétiquement les objets les plus intéressants au point de vue historico-scientifique ou artistique, soit le livretguide (Giacobini et al., 2008). Trois espaces vidéo localisés le long du parcours d'exposition leur permettent d'accéder à un choix de brefs documentaires qui présentent quelques anecdotes relatives aux objets présentées et/ou à certain événements-clés. Plus récemment, à la demande de certains visiteurs (telle qu'exprimée dans le cahier de visite ${ }^{4}$ et les retours d'information recueillis à l'issue des médiations) des fiches bilingues italien-anglais ont été disposées à côté de chaque vitrine fournissant des renseignements plus approfondis sur des thèmes d'anatomie en rapport avec les objets exposés. En définitive, le parcours d'exposition, qui mêle science, histoire et arts, tout en mettant en valeur l'esprit du lieu, se prête particulièrement bien à une lecture interdisciplinaire liant culture scientifique et culture humaniste.

\section{Le Pôle muséal}

La restauration du Musée d'Anatomie a permis l'essor d'un projet plus vaste, celui d'un nouveau pôle muséal turinois, issu d'une convention entre l'Université de Turin, la Région Piémont et la Ville de Turin, qui a conduit à l'ouverture au public de deux autres musées dans le Palais des Instituts anatomiques: le Musée des Fruits et le Musée d'Anthropologie criminelle Cesare-Lombroso, inaugurés respectivement en février 2007 et en novembre 2009.

Le Musée des Fruits (Jalla, 2008) présente une collection de plus de mille «fruits artificiels» réalisés à la fin du XIX ${ }^{e}$ siècle par Francesco Garnier Valletti, composée par des centaines de variétés de pommes, poires, pêches, abricots, prunes, raisins, dont beaucoup ont disparu. L'immersion dans le passé constitue aussi l'occasion de réfléchir sur le thème, bien actuel, de la biodiversité et de sa conservation.

Le Musée Lombroso (Bianucci et al., 2011), crée par le père fondateur de l'anthropologie criminelle, présente les collections accumulées par un chercheur mondialement connu et dont l'intérêt historico-scientifique va croissant. Mais le musée n'est pas seulement un lieu de conservation et d'exposition d'un patrimoine culturel; il est aussi un lieu de mémoire de l'œuvre de Lombroso qui, malgré ses fausses interprétations, a ouvert la porte à des préoccupations de la science sur certains problèmes 
de société, en proposant des pistes de réflexions déterminantes pour la psychiatrie et la criminologie ${ }^{5}$. Le musée se présente donc aussi comme un espace de communication qui invite le visiteur à réfléchir sur le lien entre science et société - thème émergeant à la charnière entre le XIX ${ }^{\mathrm{e}}$ et le $\mathrm{xx}^{\mathrm{e}}$ siècle, et plus que jamais d'actualité

Avec trois musées actuellement ouverts au public, qui accueillent presque deux cents visiteurs par jour, le
Pôle offre donc une vision articulée et complexe du positivisme scientifique qui, entre la fin du XIX $\mathrm{X}^{\mathrm{e}}$ et le début $\mathrm{d}$ $\mathrm{xx}^{\mathrm{e}}$ siècle, a trouvé à Turin un centre d'épanouissemen national et international (Abbot, 2008 et 2010). En valorisant un patrimoine historique, en le réinterprétant et en le rendant communicatif, ces musées offrent l'opportunité de réfléchir sur l'héritage du positivisme, ainsi que sur les nouvelles frontières de la science d'aujourd'hui et de ses rapports avec la société...

\section{NOTES}

1. De nombreuses collections universitaires font égalemen l'objet du même constat, car elles ne sont plus utilisées dans la formation initiale des étudiants que de façon exceptionnelle et anecdotique, à partir de là deux possibilités s'offrent aux institutions: soit la fermeture du musée et une deteriotic inéluctable des enes le grand public. Les stratégies de rénovation des anciens musées scientifiques (non seulement universitaires) ont fait l'objet de nombreux débats (voir, par exemple, Colin-Fromont et Lacroix 2005; en ce qui concerne les problèmes des musées d'anatomie on renvoie aux différents articles publiés dans Muséographier le corps. La Lettre de l'OCIM, $\left.{ }^{\circ} 109,2007\right)$

2. Turin était la capitale du royaume de Sardaigne, qui comprenait le Piémont, la Savoie, le comté de Nice et la Sardaigne.
3. La collection, qui rassemble plus de 200 modèles, est une des plus riches du genre. Pour leur grande majorité, ces modèles furent réalisés entre les dernières décennies du $\mathrm{XvIII}^{\mathrm{e}}$ et la moitié du XIX ${ }^{e}$ siècle à Turin, Florence et Naples.

4. L'étude du livre des visiteurs démontre que presque le $10 \%$ des personnes demandaient des renseignements anatomiques plus approfondis.

5. À l'inverse de l'école dite classique, Lombroso et ses élèves se sont efforcés de montrer que l'individu délictueux était entièrement sous contrôle de sa nature, de son état psychologique et de son environnement social, et donc, que pas ou peu responsable de ses actes, il méritait moins d'être emprisonné que soigné

\section{RÉ FÉRENCES BIB LIOGR A PHIQUES}

Аввот, А., «Hidden Treasures. Turin's Anatomy Museum», Nature, vol. 455, 2008, p. 736

Аввот, A., «Turin’s Criminology Museum », Nature, vol. 463, 2010 p. 300.

Avataneo, L. et Montaldo, S., "La Città della scienza al Valentino» in Giacobini, G. (dir.), La Memoria della scienza. Musei e collezioni dell'Università di Torino, Turin, CRT, 2003, p. 89-96.

Bianucci, P., Cilli, C., Giacobini, G., Malerba, G. et MonTAldo, S., Il Museo di Antropologia criminale Cesare Lombroso dell'Università di Torino, Turin, Edizioni Libreria Cortina, 2011.

Caputi, F., Spaziante, R., De Divitis, E. et Nashold, B. S., «Luigi Rolando and his Pioneering Efforts to Relate Structure to Function in the Nervous System ", Journal of Neurosurgery, vol. 83, 1995, p. 933-937. 
Colin-Fromont, C. et Lacroix, J.-L. (dir.), Muséums en rénovation. Les sciences de la terre et l'anatomie comparée face aux publics, Dijon, Paris, OCIM, MNHN, 2005.

DinI, A., Luigi Rolando. Saggio sopra la vera struttura del cervello dell'uomo e degli animali e sopra le funzioni del sistema nervoso, Florence, Giunti, 2001

Giacobini, G., Cilli, C. et Malerba, G., Il Museo di Anatomia
Umana Luigi Rolando dell'Università di Torino, Turin, Galleria del Libro, 2008.

Jalla, D., Il Museo della Frutta Francesco Garnier Valletti, Turin Galleria del Libro, 2008.

Muséographier le corps. Patrimoine des Universités. La Lettre de l'OCIM, $\mathrm{n}^{\circ} 109,2007$. 
Résumés - Abstracts

Scholarly museums for the modern age: Turin's Museum of Anatomy rises to the challenge

The University of Turin's Museum of Human Anatomy, founded in 1739, was transferred in 1898 to the building it still occupies, which is notable for its monumental style of architecture. It was recently restored to the atmosphere of its day, but during the project, careful consideration was given to possibilities for transforming this scholarly establishment into a communicational museum. It is one of those included in Turin's new museums project, alongside the Museum of Fruit and the Cesare Lombroso Museum of Forensic Anthropology. All three museums, which have about 200 visitors a year, offer valuable insights into scientific positivism, which was particularly influential in Turin.

Keywords: museum, human anatomy, restoration, communication, positivism.

Jean-Paul Lafrance, La révolution peut-elle être gazouillée?

Peut-on parler d'une révolution 2.0 dans le cas de l'utilisation de Twitter et des réseaux sociaux numériques pendant la révolte des populations arables dans la première moitié de 2011 ? Faut-il plutôt analyser le rôle et l'importance des outils de communication dans le processus de réveil des populations longtemps maintenues au silence par des régimes autoritaires?

Mots-clés: révolution, Twitter, Facebook, réseaux sociaux numériques, engagement, révolte arabe.

\section{Are tweets the stuff of revolution?}

Does the use of Twitter and social networks sites during the Arab revolts in early 2011 amount to a revolution 2.0? Or would it be more relevant to analyse the role and importance of communication tools generally in the awakening of populations silenced for so many years by authoritarian regimes?

Keywords: Revolution 2.0, Twitter, Facebook, social networks sites, commitment, Arab revolt.

Lina ZAKHOUR, Printemps arabe: de l'imaginaire au réel. Les moyens d'information et de communication font la révolution

Au vu de l'éclosion soudaine de ce «printemps arabe» qui a essaimé de pays en pays, cet article montre comment, à l'ère de la transparence et de la simultanéité, les moyens d'information et de communication ont créé un champ d'action 


\section{Hermès 61}

\section{Les musées au prisme}

de la communication

Numéro coordonné par Paul Rasse et Yves Girault Supervisé par Monique Veaute

\section{Sommaire}

Paul Rasse et Yves Girault

Introduction

Sélection bibliographique.

I. Les transformations de l'institution

Dominique Poulot

Le temps des musées et le temps du patrimoine.

André Desvallées et François Mairesse

L'organisation des musées: une évolution difficile.

Jean Davallon

Le pouvoir sémiotique de l'espace.

Serge Chaumier

Les écritures de l'exposition.

Jacqueline Eidelman et Anne Jonchery

Sociologie de la démocratisation des musées

Annie Van-Praët

L'image du musée dans le cinéma de fiction (encadré)

Valérie Schafer et Benjamin Thierry

Le mariage de raison du musée d'art et du Web (encadré) 
P. R.

Succès universel de l'institution muséale et des grandes expositions blockbuster.

II. Les enjeux esthétiques du musée dans le jeu de l'art contemporain

\section{Boris Groys}

Le musée pour l'installation d'art contemporain ...

Paul Rasse

Le musée protagoniste de l'art contemporain.

Christine Bernier

L'art contemporain, Internet et le musée

Joseph R. Moukarzel

Du musée-écrin au musée-objet

Patrick Barrer

Des foires-musées aux musées du marché (encadré)

Bernard de Montferrand

Les FRAC: une autre manière de rendre la culture accessible (encadré).

Brigitte Chapelain

De nouvelles médiations numériques au service de la culture augmentée (encadré)

III. Le musée de société, débats sur les cultures du monde

\section{Michel Côté}

Les musées de société : le point de bascule ...........................................................................................................

Pascal Griset et Léonard Laborie

D'entreprise ou de société? Deux opérateurs «historiques» et leurs musées, EDF et Orange.

Patrice de la Broise

La muséologie au défi d'une patrimonialisation post-industrielle

Nancy L. Green

Construire une collection, représenter l'immigration: la Cité nationale de l'Histoire de l'immigration..

Marie-Sylvie Poli et Linda Idjéraoui-Ravez

Des musées et des expositions dans le débat sur l'immigration en France...

Peter Brown

Les Musées du Pacifique: un autre regard sur le monde (encadré) 
Fabienne Galangau-Quérat, Anne Nivart et Anne Jonchery

Les musées en Namibie au cour d'une société en mutation

Pascal Dayez-Burgeon

Un musée subliminal: le Musée africain de Tervuren (encadré).

IV. Entre vulgarisation et débat public, les stratégies des musées de science en question

Yves Girault et Grégoire Molinatti

Comment les musées et centres de sciences s'exposent aux controverses socioscientifiques.

Joëlle Le Marec

L'environnement et la participation au musée: différentes expressions culturelles des sciences..

María Isabel Orellana Rivera

Science, contexte politique et musées en Amérique latine

Claudie Haigneré

Universcience (entretien)

Morgan Meyer et Peter Schüßler

Le laboratoire "en verre": exposer la science en action au musée (encadré)

Francine Boillot

L'interaction sociale des musées des sciences de la vie: une mission amnésique ou impossible? (encadré)

Giacomo Giacobini, Cristina Cilli et Giancarla Malerba

Que faire des musées de savants? Le défi du Musée d'Anatomie de Turin

Varia

Jean-Paul Lafrance

La révolution peut-elle être gazouillée?

Lina Zakhour

Printemps Arabe: de l'imaginaire au réel.

Hommages

Paul Baran (1926-2011)

(par Martin Sobieszczanski)

Lectures 


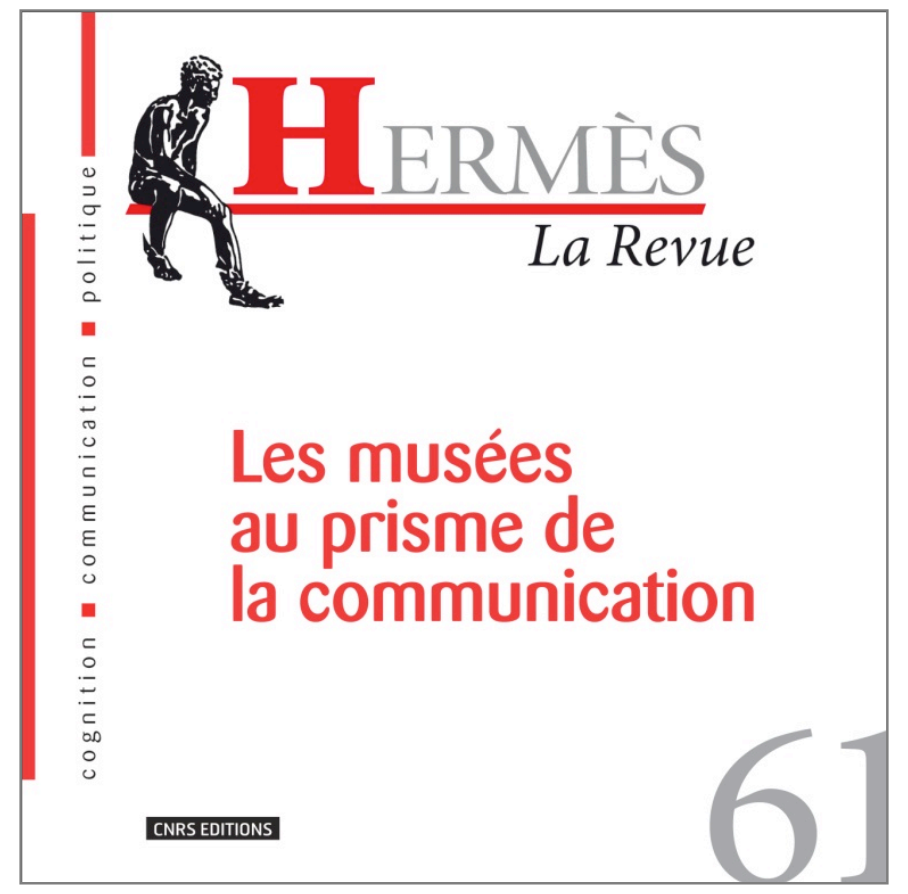

\section{Vient de paraître}

Numéro coordonné par

Paul RASSE

et

Yves GIRAULT

Supervisé par

Monique VEAUTE

Jusqu'aux années 1950, le musée faisait partie des institutions culturelles établies, s'inscrivant dans l'héritage $\mathrm{du} \mathrm{XIX}^{\mathrm{e}}$ siècle et la tradition des collectionneurs. Avec la démocratisation et le développement des industries culturelles, la situation a radicalement changé, comme en témoignent le nombre, le rôle et la visibilité de ces établissements. Dépassant les missions de conservation et de connaissance savante, la communication en direction du grand public a remis en jeu la dynamique du musée, pour en faire aujourd'hui une institution phare, rayonnant sur la cité.

Le musée, comme univers symbolique de premier plan, est désormais un prisme où se réfractent les grandes controverses. On y discerne les débats qui animent la culture sur les choix esthétiques, le sens de l'histoire, la mémoire et les processus de patrimonialisation, sur les rapports et les conflits interculturels, comme sur les mutations scientifiques et techniques. Quels sont les enjeux, les dynamiques, les dérives qui soustendent cet essor sans précédent?

Ce numéro de la revue Hermès s'intéresse à ces transformations de l'institution muséale, entre la culture, la politique, la communication, les sciences et la société. Gardant à l'esprit les innovations et succès contemporains que sont, entre autres, le Louvre, le Centre Pompidou, le Muséum national d'Histoire naturelle, le Musée du Quai Branly, la Tate Modern, le MoMA, le Capital Museum de Pékin, le Museo Nacional de Antropología de Mexico, ce dossier propose un panorama pluriel et transversal sur cette institution emblématique. Le musée, fait de culture total, sélectionne, légitime, donne à voir et à savoir au public et multiplie les traces que notre époque léguera aux générations futures.

Sommaire et résumés français/anglais en

ligne :http://www.iscc.cnrs.fr/spip.php?article1515

Revue Hermès $n^{\circ} 61$

Les musées au prisme de la communication ISSN : 0767-9513

Prix : $25 €$
CNRS Éditions - Service commercial 15 , rue Malebranche -75005 Paris 0153102700 - sabine.lavaud@cnrseditions.fr Achat en ligne : http://www.cnrseditions.fr/ 


\section{LES MUSÉES AU PRISME DE LA COMMUNICATION}

Monique Veaute - Avant-propos - Éloge de la diversité

Paul RASSE et Yves GiRault - Introduction. Regard sur les arts, les sciences et les cultures en mouvement, à travers les débats qui agitent l'institution muséale...

\section{Les transformations de l'institution}

Dominique Poulot - Le temps des musées et le temps du patrimoine André Desvallées et François MaIResse - L'organisation des musées : une évolution difficile

Jean Davallon - Le pouvoir sémiotique de l'espace : vers une nouvelle conception de l'exposition

Serge Chaumier - Les écritures de l'exposition Jacqueline EIDELMAN et Anne JonCHERY - Sociologie de la démocratisation des musées Annie VAN-PraËt - L'image du musée dans le cinéma de fiction (encadré) Succès universel de l'institution muséale et des grandes expositions blockbuster

\section{Les enjeux esthétiques du musée dans le jeu de l'art contemporain}

Boris GroYs - Le musée pour l'installation d'art contemporain

Paul RASSE - Le musée protagoniste de l'art contemporain

Christine BERNIER - L'art contemporain, Internet et le musée Joseph MouKaRzel - Du musée-écrin au musée-objet

Patrick BARRER - Des foires-musées aux musées du marché (encadré)

Bernard de MontFerRAND - Les FRAC: une autre manière de rendre la culture accessible (encadré)

Valérie Schafer et Benjamin ThierRy - Le mariage de raison du musée d'art et du Web (encadré)

Brigitte Chapelain - De nouvelles médiations numériques au service de la culture augmentée (encadré)

\section{Le musée de société, débats sur les cultures du monde}

Michel CôtÉ - Les musées de société : le point de bascule

Pascal GRISET et Léonard LABORIE - D'entreprise ou de société ? Deux opérateurs " historiques 》 et leurs musées, EDF et Orange

Patrice DE LA BRoISE - La muséologie au défi d'une patrimonialisation post-industrielle. Le cas du bassin minier Nord - Pas-de-Calais

Nancy L. Green - Construire une collection, représenter l'immigration : la Cité nationale de l'Histoire de l'immigration

Marie-Sylvie Poli et Linda IDJéraoul-Ravez - Des musées et des expositions dans le débat sur l'immigration en France

Peter Brown - Les musées du Pacifique : un autre regard sur le monde ? (encadré) 
Fabienne Galangau-QuÉrat, Anne Nivart et Anne Jonchery - Les musées en Namibie au cœur d'une société en mutation

Pascal DAyez-BuRgeon - Un musée subliminal : le Musée africain de Tervuren (encadré)

IV. Entre vulgarisation et débat public, les stratégies des musées de science en question

Yves Girault et Grégoire Molinatti - Comment les musées et centre de sciences s'exposent aux controverses socioscientifiques

Joëlle LE MAREC - L'environnement et la participation au musée : différentes expressions culturelles des sciences

María Isabel Orellana Rivera - Science, contexte politique et musées en Amérique latine

Claudie Haigneré - Les enjeux de la création d'Universcience (entretien)

Morgan MeYer et Peter SchüßLER - Le laboratoire « en verre » : exposer la science en action au musée (encadré)

Francine BollLot - L'interaction sociale des musées des sciences de la vie : une mission amnésique ou impossible ? (encadré)

Giacomo Giacobinı, Cristina CiLl et Giancarla Malerba - Que faire des musées de savants ? Le défi du Musée d'Anatomie de Turin

Postface

Dominique Wolton -- Les musées. Trois questions

- Varia

Jean-Paul LAFRANCE, La révolution peut-elle être gazouillée?

Lina ZaKHOUR, Printemps Arabe : de l'imaginaire au réel.

- Hommage

Paul Baran (1926-2011), par Marcin SobIESzCZANSKI

- Lectures 